\author{
Черняк А.В. • Савушкина О.И.' • Пашкова Т.Л. • Крюков Е.В. ${ }^{2}$
}

Черняк Александр Владимирович канд. мед. наук, заведующий лабораторией функциональных и ультразвуковых методов исследования'; ORCID: https://orcid. org/0000-0002-2001-5504

115682, г. Москва, Ореховый бульвар, 28, Российская Федерация. Тел.: +7 (917) 5500634 E-mail: achi2000@mail.ru

Савушкина Ольга Игоревна - канд. биол. наук, заведующая отделением исследований функции внешнего дыхания Центра функциональнодиагностических исследований ${ }^{2}$ ORCID https://orcid.org/0000-0002-7486-4990. E-mail: olga-savushkina@yandex.ru

Пашкова Татьяна Леонидовна - канд. мед. наук, ст. науч. сотр., лаборатория функциональных и ультразвуковых методов исследования'.

E-mail: tanya.pashkova.33@mail.ru

Крюков Евгений Владимирович д-р мед. наук, профессор, членкорреспондент PAH, начальник²; ORCID: https://orcid.org/0000-0002-8396-1936.

E-mail: evgeniy.md@mail.ru

ФГБУ «Научно-исследовательский институт пульмонологии» ФМБА России; 115682 , г. Москва, Ореховый бульвар, 28, Российская Федерация

${ }^{2}$ ФГБУ «Главный военный клинический госпиталь имени академика Н.Н. Бурденко» Минобороны России; 105094, г. Москва, Госпитальная пл., 3 , Российская Федерация
Актуальность. Дисфункция малых дыхательных путей (ДМДП) - функциональный признак хронической обструктивной болезни легких (ХОБЛ). Однако распространенность ДМДП и ее роль в патофизиологических процессах при ХОБЛ изучена недостаточно.

Цель - оценить распространенность ДМДП у пациентов с ХОБЛ с помощью различных методов функциональной диагностики: спирометрии, бодиплетизмографии, импульсной осциллометрии (ИОС).

Материал и методы. Дизайн исследования обсервационное поперечное. Спирометрия, бодиплетизмография и ИОС выполнены 132 пациентам с ХОБЛ, находящимся в состоянии ремиссии и получающим стандартное лечение ХОБЛ. Заключение о наличии ДМдП делали на основании выявления одного из следующих критериев или их комбинации: 1) по спирометрии: разница между жизненной емкостью легких (ЖЕЛ) и форсированной жизненной емкостью легких (ФЖЕЛ) > 10\%; 2) наличие воздушных ловушек по данным бодиплетизмографии; 3) наличие частотной зависимости резистивного сопротивления при частоте осцилляций 5 и 20 Гц (R5 - R20>0,07 кПа× с/л) по данным ИОС.

Результаты. Среднее значение объема форсированного выдоха за 1 секунду (ОФВ1) составило $42,9 \%$ должного. В зависимости от степени тяжести обструкции пациенты были разделены на 4 группы: у 7 пациентов (1-я группа) обструктивные нарушения соответствовали стадии GOLD 1, у 37 (2-я группа) - стадии GOLD 2, у 49 (3-я группа) - стадии GOLD 3 и у 39 (4-я группа) - стадии GOLD 4. ДМДП выявлена у 96\% пациентов с ХОБЛ и у 100\% больных с тяжелой обструкцией (стадии GOLD 3-4). C помощью спирометрии дМдП определена только у 67\% пациентов с ХОБЛ; бодиплетизмографии - у 75\% пациентов с ХОБЛ (у пациентов со стадией GOLD 3 и 4 - у 88 и 97\% соответственно); ИОС - у 94\% пациентов с ХОБЛ (у пациентов со стадией GOLD 3-4 - в 100\% случаев).

Заключение. Для пациентов с ХОБЛ по мере нарастания обструктивных нарушений легочной вентиляции характерно прогрессирующее увеличение дисфункции периферических отделов дыхательных путей. Импульсная осциллометрия представляется наиболее эффективным методом диагностики дисфункции периферических отделов дыхательных путей, так как с ее помощью ДМдП была выявлена у 94\% пациентов с ХОБЛ в целом по группе и у 100\% пациентов с тяжелой и очень тяжелой степенью обструктивных нарушений.

Ключевые слова: дисфункция малых дыхательных путей, хроническая обструктивная болезнь легких, импульсная осциллометрия, спирометрия, бодиплетизмография

Для цитирования: Черняк $A B$, Савушкина $O И$, Пашкова ТЛ, Крюков ЕВ. Диагностика дисфункции малых дыхательных путей у пациентов с хронической обструктивной болезнью легких. Альманах клинической медицины. 2020;48(5):307-15. doi: 10.18786/2072-0505-2020-48-019.

Поступила 29.10.2019; доработана 19.11.2019; принята к публикации 01.04.2020; опубликована онлайн 10.04.2020 
$\mathrm{X}$ роническая обструктивная болезнь легких (ХОБЛ) - распространенное заболевание, представляющее глобальную медико-социальную и экономическую нах $[1,2]$. По данным официальной статистики, в России число больных ХОБЛ насчитывает приблизительно 3 млн, тогда как результаты эпидемиологического исследования GARD говорят о том, что фактически этот показатель выше в 9,3 раза [3]. В 2006 г. для диагностики и определения степени тяжести ХОБЛ согласно международным рекомендациям (Глобальная инициатива по хронической обструктивной болезни легких, англ. Global Initiative for Chronic Obstructive Lung Disease - GOLD) необходимо было провести спирометрические измерения с определением объема форсированного выдоха в 1 секунду $\left(\mathrm{O}_{1}\right)$ и форсированной жизненной емкости легких (ФЖЕЛ) [4]. Сегодня подход к определению степени тяжести ХОБЛ изменился $[2,5]$. Как и ранее, для подтверждения диагноза ХОБЛ отношение $\mathrm{OФB}_{1}$ к ФЖЕЛ $\left(\mathrm{OФВ}_{1} /\right.$ ФЕЛ) после максимальной бронходилатации должно быть менее 0,7 [2, $4,5]$. Но для оценки степени тяжести заболевания помимо ОФВ 1 современный диагностический алгоритм учитывает историю предыдущих обострений и клинические симптомы: одышку (измеренную с помощью модифицированной шкалы Совета по медицинским исследованиям $\mathrm{mMRC})$ и состояние здоровья (измеряемое с помощью шкалы CAT) $[2,5]$.

У пациентов с ХОБЛ ключевым местом обструкции становятся малые (или периферические) дыхательные пути (МДП), то есть дыхательные пути с диаметром менее 2 мм, которые начинаются уже с 5-6-й генерации и включают в себя терминальные и респираторные бронхиолы, альвеолярные ходы и альвеолярные мешочки [6-8]. Дисфункция малых дыхательных путей (ДМДП), обусловленная их воспалением, отеком и спазмом, считается характерным функциональным признаком ХОБЛ $[9,10]$. Были выявлены корреляционные связи между ДМДП и восприятием одышки, качеством жизни у пациентов с ХОБЛ [11], однако ее распространенность и роль в патофизиологических процессах при ХОБЛ изучена недостаточно. Для оценки функции МДП у пациентов с ХОБЛ в клинической практике были предложены различные неинвазивные методы функциональной диагностики, такие как спирометрия, бодиплетизмография, импульсная осциллометрия (ИОС), вымывание азота при множественном дыхании [12-15].
Цель данной работы - оценить распространенность ДМДП у пациентов с ХОБЛ с разной степенью тяжести бронхиальной обструкции при помощи различных методов функциональной диагностики (спирометрии, бодиплетизмографии, ИОС).

\section{Материал и методы}

В обсервационное поперечное исследование были включены 132 пациента с ХОБЛ - 127 (96\%) мужчин и 5 (4\%) женщин, медиана возраста 66 (от 61 до 71) лет, проходивших лечение и наблюдение в 2010-2017 гг. в двух медицинских учреждениях г. Москвы (ФГБУ «НИИ пульмонологии» ФМБА России и ФГБУ «Главный военный клинический госпиталь имени академика Н.Н. Бурденко» Минобороны России). На момент включения в исследование 82 (62\%) пациента продолжали курить и 50 (38\%) бросили. Диагноз ХОБЛ был установлен в соответствии с клиническими рекомендациями Российского респираторного общества (PРО) [5]. Все пациенты были в состоянии ремиссии и получали стандартное лечение ХОБЛ в соответствии с рекомендациями РPO [5].

Всемпациентамбыли выполнены комплексное исследование функции внешнего дыхания (форсированная спирометрия, бодиплетизмография, измерение диффузионной способности легких) и ИОС. Все функциональные исследования проводили в первой половине дня, после применения бронходилатационной терапии. Исследования проводились на установках MasterScreen Body и MasterScreen IOS (Viasys Healthcare, Германия). Спирометрия, бодиплетизмография и диффузионный тест выполнены с соблюдением стандартов качества исследований Американского торакального общества (АТО) и Европейского респираторного общества (ЕРО) [16-18]. ИОС проводили в соответствии с рекомендациями H.J. Smith и соавт. [19]. Диффузионную способность легких $\left(\mathrm{DL}_{\mathrm{CO}}\right)$ оценивали при однократном вдохе газовой смеси, содержащей монооксид углерода (CO), с задержкой дыхания [18].

Анализировали следующие параметры:

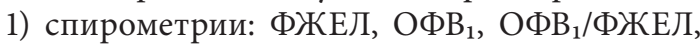
ОФВ 1 ЖЖЕЛ (ЖЕЛ - Жизненная емкость легких), среднюю объемную скорость на участке кривой «поток - объем форсированного выдоха» между 25 и 75\% ФЖЕЛ ( $\left.\mathrm{COC}_{25-75}\right)$;

2) бодиплетизмографии: ЖЕЛ, общую емкость легких (ОЕЛ), остаточный объем легких (ООЛ) и его долю в ОЕЛ (ООЛ/ОЕЛ), внутригрудной объем газа (ВГО), общее бронхиальное сопротивление $\left(\mathrm{Raw}_{\text {общ. }}\right)$ и бронхиальное сопротивление 
между потоками 0,5 л/с на вдохе и выдохе $\left(\operatorname{Raw}_{0,5}\right.$ : отражает прежде всего проходимость центральных дыхательных путей);

3) диффузионного теста: $\mathrm{DL}_{\mathrm{CO}}$ и отношение $\mathrm{DL}_{\mathrm{CO}}$ к альвеолярному объему $(\mathrm{Va})\left(\mathrm{K}_{\mathrm{CO}}\right.$ : отражает деструкцию паренхимы легких [20]);

4) ИОС: дыхательный импеданс при частоте осцилляций 5 Гц (Z5); резистивное сопротивление при частоте осцилляций 5 и 20 Гц (R5 и R20 соответственно); реактивное сопротивление при частоте осцилляций 5 Гц (X5), частотной зависимости R: относительной (R5 - R20)/R5) и абсолютной (R5 - R20); резонансной частоты $\left(\mathrm{f}_{\text {res }}\right)$; площади реактанса (AX).

При анализе показателей, полученных в результате комплексного исследования функции внешнего дыхания, использовали должные значения для общей популяции, которые рассчитывали по формулам Европейского объединения угля и стали (ECSC, 1993) [21] с учетом антропометрических характеристик (пол, возраст и рост). Результаты выражали в процентах от должного значения (\% долж.): полученное значение / должное значение $\times 100 \%$. Отклонения от нормы параметров ИОС определяли с помощью классического алгоритма: по изменению базовых показателей R5 и X5 [22]. Перед исследованием функции внешнего дыхания измеряли рост и массу тела (без обуви и верхней одежды).

Степень тяжести ограничения воздушного потока оценивали в соответствии с международными рекомендациями по диагностике и ведению больных ХОБЛ (GOLD) следующим образом: $\mathrm{OФB}_{1}$ составляет $80 \%$ долж. и более - легкие нарушения (GOLD 1), от 50 до 79\% долж. - умеренные (GOLD 2), от 30 до 49\% долж. - тяжелые (GOLD 3), менее 30\% долж. - крайне тяжелые (GOLD 4) [2].

ДМДП приводит к сужению их просвета, вплоть до полного закрытия, следствием чего является задержка воздуха в альвеолах («воздушная ловушка») и неравномерное распределение вентиляции. Именно поэтому тесты, которые позволяют оценить эти изменения, могут быть полезными в клинической практике для выявления и количественной оценки ДМДП. В нашей работе заключение о наличии ДМДП было сделано на основании выявления одного из следующих критериев или их комбинации $[23,24]$ :

1) относительный прирост ЖЕЛ-ФЖЕЛ к ЖЕЛ (ЖЕЛ - ФЖЕЛ) > 10\%;

2) наличие воздушных ловушек: ООЛ > долж. $+1,64 \mathrm{RSD}$ и ООЛ/ОЕЛ > долж. $+1,64$ $\mathrm{RSD}$, где RSD - стандартное отклонение;

3) $\mathrm{R} 5$ - R20 >0,07 кПа $\times$ c/л.
Статистическая обработка результатов выполнена методами описательной статистики с применением прикладного пакета программ STATISTICA 10.0 (StatSoft Inc., США). Для оценки нормальности распределения переменных использовали критерий Колмогорова - Смирнова с поправкой Лиллиефорса. Данные представлены как медианы (Mе) (1-й квартиль; 3-й квартиль) для непрерывных переменных с ненормальным распределением. Количество пациентов (n) использовалось для категориальных переменных. Категориальные переменные сравнивались с помощью точного критерия Фишера, непрерывные переменные - непараметрического критерия Краскела - Уоллиса с последующим попарным сравнением групп с помощью U-критерия Манна - Уитни. Корреляционный анализ проводили с использованием ранговой корреляции Спирмена. Различия считались статистически значимыми при $\mathrm{p}<0,05$.

\section{Результаты}

В группе обследованных преобладали больные с тяжелой и очень тяжелой степенью бронхиальной обструкции, среднее значение ОФВ

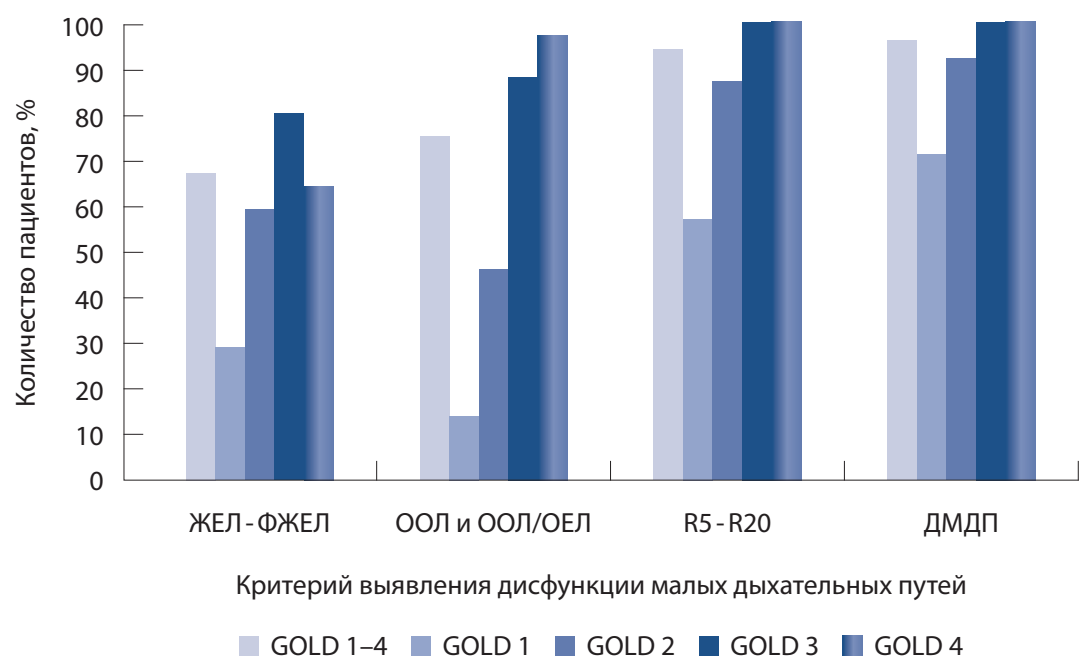

Рис. 1. Частота встречаемости дисфункции малых дыхательных путей у пациентов с хронической обструктивной болезнью легких в зависимости от метода диагностики в целом по группе и при разной степени обструкции; GOLD 1-4 - все пациенты,

GOLD 1 - пациенты с объемом форсированного выдоха за 1 секунду (ОФВ,) 80\% должного значения (долж.) и более, GOLD 2 - пациенты с OФВ в в диапазоне 50-79\% долж., GOLD 3 пациенты с ОФВ, в диапазоне 30-49\% долж., GOLD 4 - пациенты с OФВ, менее 30\% долж., R5 - R20 - частотная зависимость: разность между резистивным сопротивлением при частоте осцилляций 5 и 20 Гц более 0,07 кПахс/л; ДМДП - выявление дисфункции малых дыхательных путей с помощью одного критерия или их комбинации, ЖЕЛ-ФЖЕЛ - разность между жизненной емкостью легких (ЖЕЛ) и форсированной жизненной емкостью легких (ФЖЕЛ) более 10\%, ООЛ и ООЛ/ОЕЛ - остаточный объем легких (ООЛ) больше долж. плюс 1,64 RSD и отношение ООЛ к ОЕЛ больше долж. плюс 1,64 RSD, где RSD - стандартное отклонение 
Характеристика пациентов, показатели легочной вентиляции и легочного газообмена, импульсной осциллометрии у пациентов с хронической обструктивной болезнью легких

\begin{tabular}{|c|c|c|c|c|c|}
\hline Показатель & GOLD 1-4 (n=132) & GOLD $1(n=7)$ & GOLD $2(n=37)$ & GOLD $3(n=49)$ & GOLD $4(n=39)$ \\
\hline Возраст, годы & $66(61-71)$ & $62(52-78)$ & $66(61-71)$ & $69(63-73)$ & $64(58-70)^{\ddagger}$ \\
\hline Пол (м/ж) & $127 / 5$ & $6 / 1$ & $35 / 2$ & $47 / 2$ & $39 / 0$ \\
\hline Курение (да/экс) & $82 / 50$ & $5 / 2$ & $26 / 11$ & $28 / 21$ & $23 / 16$ \\
\hline Рост, сM & $172(168-177)$ & $170(169-179)$ & $173(170-178)$ & $171(168-175)$ & $173(168-178)$ \\
\hline ИМТ (кг/M²) & $26(23-30)$ & $26(23-28)$ & $30(24-34)$ & $27(24-30)$ & $25(21-27)^{+, \neq}$ \\
\hline ЖЕЛ, \% долж. & 85 (71-99) & $117(101-127)$ & $97(88-107)^{*}$ & $82(72-91)^{*},+$ & $70(65-80)^{*},+, \neq$ \\
\hline ФЖЕЛ, \% дОЛЖ. & $72(61-85)$ & $103(97-130)$ & $85(79-95)^{*}$ & $69(60-76)^{*},+$ & $62(52-71)^{*},+, \neq$ \\
\hline ЖЕЛ-ФЖЕЛ, Л & $0,52(0,23-0,78)$ & $0,11(0,03-0,70)$ & $0,52(0,21-0,78)$ & $0,61(0,30-0,77)^{*},+$ & $0,46(0,22-0,75)$ \\
\hline ЖЕЛ - ФЖЕЛ > 10\% (Да/НеТ) & $88 / 44$ & $2 / 5$ & $22 / 15$ & $39 / 10^{*}+$ & $25 / 14$ \\
\hline ОФВ $1, \%$ долж. & $40(28-54)$ & $84(82-95)$ & $60(53-65)^{*}$ & $39(33-43)^{*},+$ & $25(22-27)^{*},+, \neq$ \\
\hline ОФВ $/$ ФЖЕЛ, \% & $40(32-52)$ & $62(58-67)$ & $54(47-61)^{*}$ & $39(33-44)^{*},+$ & $31(25-36)^{*},+\neq$ \\
\hline $\mathrm{MOC}_{50,} \%$ долж. & $12(8-20)$ & $40(35-47)$ & $23(18-30)^{*}$ & $12(10-15)^{*},+$ & $7(5-8)^{*}+, \neq \neq$ \\
\hline $\mathrm{COC}_{25-75,}$ \% долж. & $14(9-22)$ & $39(35-51)$ & $23(21-31)^{*}$ & $13(11-17)^{*},+$ & $8(6-10)^{*,+, \neq}$ \\
\hline ОЕЛ, \% долж. & $122(108-133)$ & $120(114-125)$ & $116(104-125)$ & $116(104-127)$ & $133(124-146)^{*},+, \neq$ \\
\hline ВГО, \% долж. & $150(128-185)$ & $132(110-146)$ & $129(107-147)$ & $144(129-155)^{\dagger}$ & $195(172-217)^{*,+, \neq}$ \\
\hline оОл, \% долж. & $181(145-222)$ & $135(121-151)$ & $147(121-173)$ & $176(151-195)^{*,+}$ & $251(218-279)^{*,+, \neq}$ \\
\hline ООЛ/ОЕЛ, \% & $57(50-64)$ & $44(38-45)$ & $48(43-51)^{*}$ & $57(55-61)^{*},+$ & $68(62-71)^{*},+, \neq$ \\
\hline Наличие воздушных ловушек (да/нет) & $99 / 33$ & $1 / 6$ & $17 / 20$ & $43 / 6^{*}+$ & $38 / 1^{*},+$ \\
\hline $\operatorname{Raw}_{\text {общ, }}, \mathrm{k} П \mathrm{a} \times \mathrm{c} / л$ & $0,69(0,49-0,90)$ & $0,27(0,14-0,49)$ & $0,45(0,30-0,60)$ & $0,76(0,64-1,01)^{*},+$ & $0,81(0,59-1,15)^{*},+$ \\
\hline $\operatorname{Raw}_{0,5}, \mathrm{k} П \mathrm{a} \times \mathrm{c} / л$ & $0,34(0,26-0,44)$ & $0,27(0,22-0,44)$ & $0,26(0,23-0,34)$ & $0,39(0,29-0,47)^{\dagger}$ & $0,38(0,31-0,45)^{\dagger}$ \\
\hline $\mathrm{DL}_{\mathrm{co}}$ \% долж. & $58(46-72)$ & $73(69-80)$ & $72(66-85)$ & $59(47-71)^{+}$ & $47(37-51)^{*}, t, \neq$ \\
\hline $\mathrm{K}_{\mathrm{co}}, \%$ долж. & $71(53-94)$ & $64(53-92)$ & $81(71-102)$ & $85(62-98)$ & $50(41-64)^{+, ~}$ \\
\hline R5, \% долж. & $186(146-222)$ & $103(71-125)$ & $148(121-190)^{*}$ & $191(167-225)^{*,+}$ & $203(165-245)^{*,+}$ \\
\hline R20, \% долж. & $114(92-139)$ & $87(76-112)$ & $112(89-131)$ & $118(99-139)^{*}$ & $112(85-142)$ \\
\hline (R5-R20)/R5, \% & $46(38-53)$ & $22(9-41)$ & $38(31-44)$ & $47(42-52)^{*},+$ & $54(46-58)^{*}, t, \neq$ \\
\hline (R5-R20), кПа×c/л & $0,28(0,17-0,35)$ & $0,08(0,02-0,15)$ & $0,19(0,12-0,27)^{*}$ & $0,29(0,22-0,35)^{*},+$ & $0,33(0,26-0,39)^{*},+$ \\
\hline (R5 - R20) > 0,07 (да/нет) & $124 / 8$ & $4 / 3$ & $32 / 5$ & $49 / 0^{*},+$ & $39 / 0^{*},+$ \\
\hline X5, кПахс/л & $-0,34(-0,45-(-0,20))$ & $-0,10(-0,14-(-0,08))$ & $-0,23(-0,28-(-0,13))^{*}$ & $-0,38(-0,481-(-0,24))^{*,+}$ & $-0,46(-0,55-(-0,34))^{*},+$ \\
\hline AX, кПа/л & $2,77(1,27-4,07)$ & $0,31(0,17-1,26)$ & $1,82(0,83-2,60)^{*}$ & $3,39(2,04-4,56)^{*},+$ & $3,39(1,39-4,55)^{*},+$ \\
\hline $\mathrm{f}_{\mathrm{res}}$ ГL & $26(22-29)$ & $13(10-21)$ & $23(18-27)^{*}$ & $26(23-28)^{*,+}$ & $28(25-31)^{*,+}$ \\
\hline Дмдп (да/нет) & $127 / 5$ & $5 / 2$ & $34 / 3$ & $49 / 0$ & $39 / 0$ \\
\hline
\end{tabular}

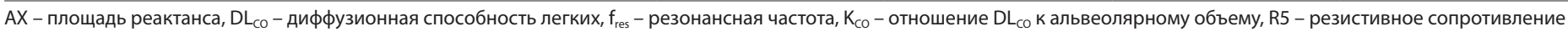

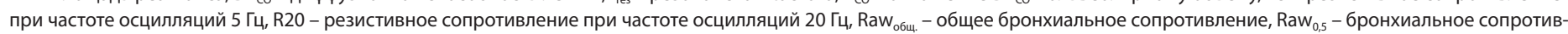

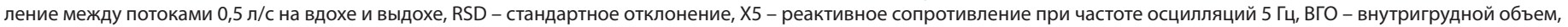

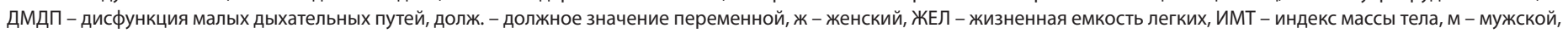

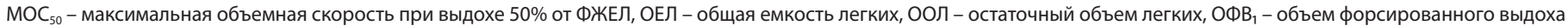
за 1 секунду, СОС $25-75$ - средняя объемная скорость выдоха на уровне 25-75\% ФЖЕЛ, ФЖЕЛ - форсированная жизненная емкость легких, экс - бывший курильщик Данные представлены как медиана (нижний - верхний квартиль) или как количество пациентов (n) для категориальных переменных * $\mathrm{p}<0,05$ при сравнении со стадией GOLD 1 ${ }^{\dagger} p<0,05$ при сравнении со стадией GOLD 2 ${ }^{\ddagger} \mathrm{p}<0,05$ при сравнении со стадией GOLD 3 


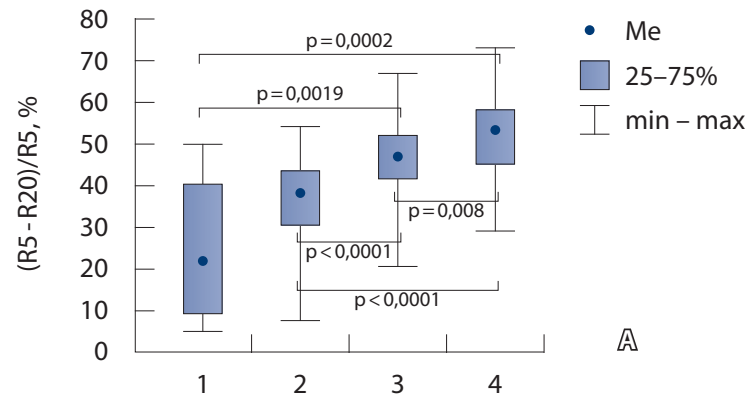

Степень обструкции, стадия GOLD

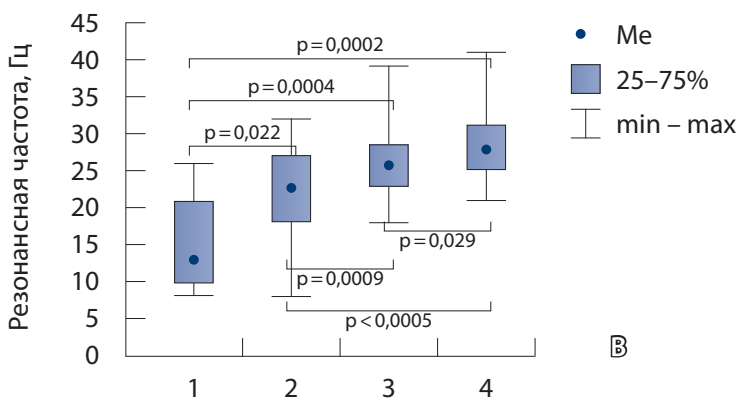

Степень обструкции, стадия GOLD

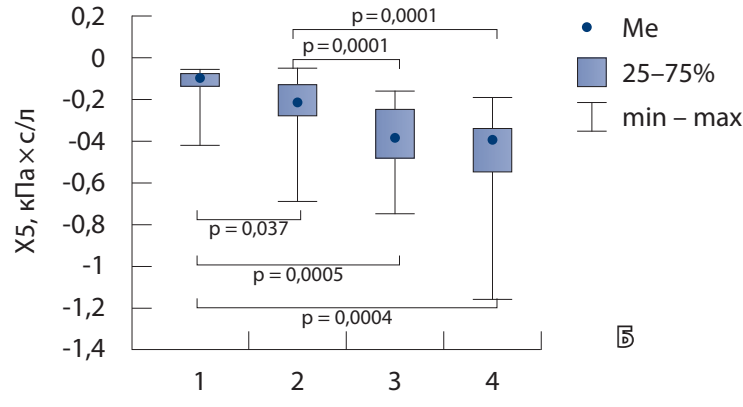

Степень обструкции, стадия GOLD

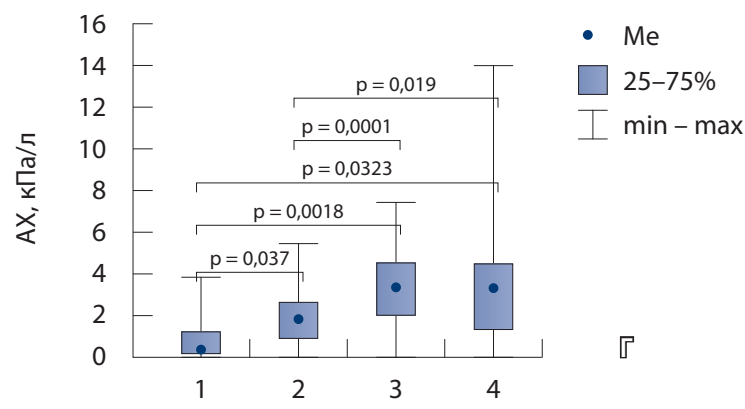

Степень обструкции, стадия GOLD

Рис. 2. Динамика показателей импульсной осциллометрии у пациентов с хронической обструктивной болезнью легких в зависимости от степени обструкции: A - (R5 - R20)/R5 - частотная зависимость: относительная разность между резистивным сопротивлением при частоте осцилляций 5 (R5) и 20 (R20) Гц, Б - X5 - реактивное сопротивление при частоте осцилляций 5 Гц, B - резонансная частота, $\Gamma$ - AX - площадь под кривой реактивного сопротивления между 5 Гц и резонансной частотой; GOLD 1 - пациенты с объемом форсированного выдоха за 1 секунду $\left(O Ф B_{1}\right)$ 80\% должного значения (долж.) и более,

GOLD 2 - пациенты с OФВ, в диапазоне 50-79\% долж., GOLD 3 - пациенты с OФВ, в диапазоне 30-49\% долж., GOLD 4 - пациенты с ОФВ 1 менее $30 \%$ долж.

составило $42,9 \pm 18,7 \%$ долж. В зависимости от степени тяжести обструкции пациенты были разделены на 4 группы: у 7 пациентов (1-я группа) обструктивные нарушения соответствовали стадии GOLD 1, у 37 (2-я группа) - стадии GOLD 2, у 49 (3-я группа) - стадии GOLD 3 и у 39 (4-я группа) - стадии GOLD 4. Характеристика пациентов, а также значения показателей легочной вентиляции, легочного газообмена и ИОС всей группы в целом и в зависимости от стадии GOLD обобщены в таблице.

Частота встречаемости ДМДП в зависимости от выбранного критерия диагностики отражена на рис. 1. Анализ данных о наличии ДМДП показал, что нарушение проходимости МДП выявлено у 96\% пациентов с ХОБЛ и увеличивается по мере нарастания обструкции: ДМДП обнаружена у 100\% больных с тяжелой и очень тяжелой обструкцией (стадии GOLD 3 и GOLD 4). В целом по группе ДМДП с помощью одного метода была выявлена у 10 (7,6\%) пациентов с ХОБЛ, двумя методами - у 50 (37,9\%) и тремя методами - у 67 (50,8\%). Основным методом диагностики ДМДП оказалась ИОС и выбранный критерий R5 - R20>0,07 кПахс/л (см. рис. 1).

Были проанализированы и другие показатели ИОС в зависимости от степени обструктивных нарушений у пациентов с ХОБЛ. По мере нарастания обструкции отмечено прогрессивное статистически значимое увеличение (R5 - R20)/R5 и $\mathrm{f}_{\text {res }}$, так же как и AX со снижением X5 (см. таблицу, рис. 2).

На рис. 3 дан график статистически значимой отрицательной корреляционной связи между $\mathrm{OФB}_{1}$ и показателями, которые были выбраны для диагностики ДМДП ( $\mathrm{R}_{\text {оол, \% долж. }}=-0,761$; $\mathrm{R}_{\text {Оол/ОЕл, \% }}=-0,848 ; \quad \mathrm{R}_{(\mathrm{R} 5-\mathrm{R} 20) / \mathrm{R} 5, \%}=-0,585 ; \quad \mathrm{R}_{\mathrm{R} 5-\mathrm{R} 20 \text {, }}$ кПахс/л $=-0,456 ; \mathrm{p}<0,0001)$, кроме разности между ЖЕЛ - ФЖЕЛ, \%. По мере нарастания обструкции увеличивается также сопротивление МДП, что приводит к выраженной частотной зависимости резистивного сопротивления (см. рис. $3 \mathrm{~B}$, Г) при 

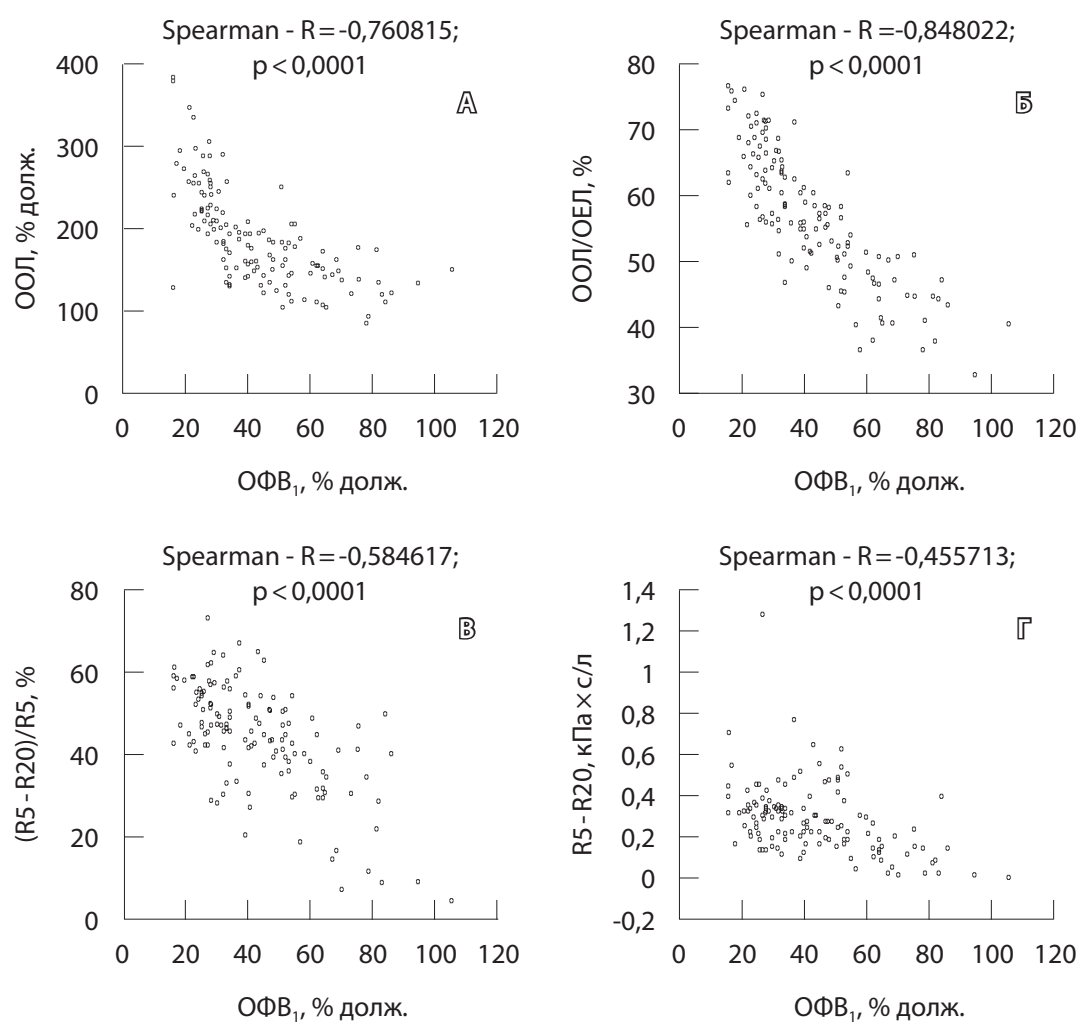

Рис. 3. График корреляционной связи между объемом форсированного выдоха за 1 секунду $\left(O Ф B_{1}\right)$ и остаточным объемом легких (ООЛ) (A), его долей в структуре общей емкости легких (ООЛ/ОЕЛ) (Б), частотной зависимостью резистивного сопротивления при частоте осцилляций 5 и 20 Гц - относительной ((R5 - R20)/R5) (B) и абсолютной (R5 - R20) (Г) у пациентов с хронической обструктивной болезнью легких

проведении ИОС и увеличению степени воздушных ловушек, о чем свидетельствует рост ООЛ и его доли в структуре общей емкости легких (см. рис. 3 А, Б) при бодиплетизмографии.

\section{Обсуждение}

В данном наблюдательном исследовании было показано, что для пациентов с ХОБЛ в стадии ремиссии по мере нарастания обструктивных нарушений легочной вентиляции характерно прогрессирующее увеличение выявления дисфункции периферических дыхательных путей, определяемой с помощью как традиционных методов исследования (спирометрия, бодиплетизмография), так и импульсной осциллометрии. У пациентов с тяжелыми обструктивными нарушениями (стадии GOLD 3-4) ДМДП была выявлена в 100\% наблюдений.

Для диагностики ДМДП используют различные функциональные методы исследования: измерение сопротивления дыхательных путей с помощью бодиплетизмографии, методом перекрытия дыхательных путей; спирометрию; измерение статических легочных объемов; исследование равномерности вентиляции с помощью метода вымывания азота при множественном дыхании, ИОС [12-15]. Выбор методик, использованных в данной работе, был обусловлен распространенностью их применения в России.

Спирометрия - метод, который применяется наиболее широко во всем мире и в нашей стране для диагностики обструктивных нарушений. Основным спирометрическим показателем, безусловно, является ОФВ 1 - интегральный показатель, отражающий сопротивления всего трахеобронхиального дерева [25-27]. Но ОФВ 1 не отражает нарушения проходимости на уровне МДП. Часто для оценки ДМДП используют другой спирометрический показатель - $\mathrm{COC}_{25-75}[15,23,27]$. Однако значение $\mathrm{COC}_{25-75}$ существенно зависит от обструкции центральных дыхательных путей и изменения объема легких, не коррелирует с выраженностью воспаления в МДП, поэтому в данной работе мы его не использовали. Мы применяли в качестве критерия наличия ДМДП разницу ЖЕЛ и ФЖЕЛ - потенциальный и довольно простой индикатор коллапса дыхательных путей во время форсированного выдоха (то есть воздушной ловушки). Увеличение этого критерия может косвенно свидетельствовать об обструкции МДП или потере эластической отдачи легких. В данном исследовании с помощью этого критерия ДМДП была выявлена только у $67 \%$ пациентов с ХОБЛ, что значительно меньше, чем при использовании других критериев. Аналогичные данные о низкой диагностической ценности этого критерия были получены и в работе Т. Perez и соавт. [23]. Это указывает на необходимость дальнейших исследований по оценке пригодности данного параметра и выработке оптимальных пороговых значений для применения его в клинической практике.

Другой выбранный нами критерий, который также отражал задержку воздуха в легких (воздушные ловушки), - комбинация ООЛ > долж. +1,64 RSD и ООЛ/ОЕЛ > долж. $+1,64$ RSD. Такой подход позволил выявить ДМДП у 75\% пациентов с ХОБЛ и был особенно эффективен у пациентов с тяжелой обструкцией (стадии GOLD 3-4), у которых частота встречаемости ДМДП составила 88 и 97\% соответственно. Мы использовали комбинацию ООЛ и его доли в структуре ОЕЛ, поскольку применение каждого из этих показателей по отдельности может приводить к ложноположительной диагностике ДМДП у пациентов с объемом легких выше среднестатистических показателей.

У большинства пациентов (94\%) мы смогли идентифицировать ДМДП с помощью критерия 
R5 - R20>0,07 кПа×c/л, то есть выраженной частотной зависимости резистивного сопротивления. R5 определяет сопротивление всех отделов дыхательных путей (и центральных, и периферических), тогда как R20 - сопротивление центральных отделов дыхательных путей, поэтому по разнице R5 - R20 судят о состоянии периферических дыхательных путей. Выбор порогового значения увеличения R5 - R20 как 0,07 кПахс/л обусловлен тем, что именно это значение является верхней границей нормы [24]. Но некоторые исследователи применяли и более низкие значения в качестве диагностического критерия - 0,03 кПахс/л [28, 29]. Сравнение полученных данных затруднено как выбором пороговых значений для выявления ДМДП, так и степенью обструктивных нарушений обследуемых пациентов с ХОБЛ. В нашей работе в целом по группе ДМДП выявили с помощью ИОС у 94\% пациентов, при этом доля пациентов с легкими обструктивными нарушениями (стадия GOLD 1) составила всего 5,3\%, а доля пациентов с тяжелыми обструктивными нарушениями (стадии GOLD 3-4) - 66,7\%. E. Crisafulli и соавт. диагностировали ДМДП у 74\% пациентов с ХОБЛ, среднее значение $\mathrm{OФB}_{1}$ в целом по группе составило 55\% долж. [30]. Эти данные согласуются с полученными нами результатами, несмотря на то что частота выявления ДМДП оказалась ниже по сравнению с нашими данными, но и ОФВ 1 в нашей популяции больных был ниже (среднее значение равно 43\% долж., медиана 40\% долж.). Кроме того, обнаружены статистически значимые отрицательные корреляционные связи между ОФВ и частотной зависимостью $\left(\mathrm{R}_{(\mathrm{R} 5-\mathrm{R} 20) / \mathrm{R} 5, \%}=-0,585\right.$;
$\left.\mathrm{R}_{\mathrm{R} 5 \text { - } 220 \text {, кПахс/л }}=-0,456 ; \mathrm{p}<0,0001\right)$, то есть у пациентов с ХОБЛ чем ниже ОФВ 1 , тем выше разность R5 - R20.

Общеизвестно, что ХОБЛ представляет собой заболевание, которое характеризуется частично обратимой обструкцией дыхательных путей [2, 5], тем не менее значительная часть пациентов с ХОБЛ может демонстрировать выраженный положительный бронходилатационный ответ $[31,32]$. В этой связи методы, позволяющие диагностировать ДМДП, могут играть важную роль в оценке эффективности проводимой терапии и выявлении тех пациентов, у которых на фоне лечения отмечается увеличение объема легких, снижение степени гиперинфляции легких и воздушной ловушки, даже в отсутствие существенной положительной динамики ОФВ 1 [33]. Внедрение таких методов в клиническую практику может способствовать прогрессу в персонализированном лечении пациентов с ХОБЛ.

\section{Заключение}

У пациентов с ХОБЛ в стадии ремиссии по мере нарастания обструктивных нарушений наблюдается прогрессирующая и возрастающая дисфункция периферических дыхательных путей. Импульсная осциллометрия представляется более эффективным методом диагностики дисфункции периферических отделов дыхательных путей по сравнению со спирометрией и бодиплетизмографией, так как с ее помощью ДМДП была выявлена у 94\% пациентов с ХОБЛ в целом по группе и у $100 \%$ пациентов с тяжелой и очень тяжелой степенью обструктивных нарушений. (๕)

\section{Дополнительная информация}

\section{Финансирование}

Работа проведена без привлечения дополнительного финансирования со стороны третьих лиц.

Конфликт интересов

Авторы декларируют отсутствие явных и потенциальных конфликтов интересов, связанных с публикацией настоящей статьи.

Участие авторов

А.В. Черняк - разработка дизайна проекта, формирование групп пациентов, набор клинического материала, анализ и интерпретация результатов, написание текста; О.И. Савушкина - разработка дизайна проекта, формирование групп пациентов, набор клинического материала, анализ и интерпретация результатов, написание и редактирование текста; Т.Л. Пашкова - набор клинического материала, анализ и интерпретация результатов, редактирование текста; Е.В. Крюков концепция и дизайн статьи, редактирование текста, утверждение итогового варианта текста рукописи. Все авторы внесли существенный вклад в проведение исследования и подготовку статьи, прочли и одобрили финальную версию перед публикацией

\section{Литература / References}

1.Всемирная организация здравоохранения. Хроническая обструктивная болезнь легких (ХОБЛ) [Интернет]. Доступно на: https://www.who.int/news-room/fact-sheets/ detail/chronic-obstructive-pulmonary-disease-(copd).[World Health Organization. Chronic obstructive pulmonary disease
(COPD) [Internet]. Accessed: October, 2019. Available from: https://www.who.int/newsroom/fact-sheets/detail/chronic-obstructive-pulmonary-disease-(copd).]

2. Global Initiative for Chronic Obstructive Lung Disease. Global Strategy for the Diagnosis, Management and Prevention of Chron- ic Obstructive Pulmonary Disease (2019 report) [Internet]. Accessed: October, 2019. Available from: https://goldcopd.org/wp-content/uploads/2018/11/GOLD-2019-v1.7-FINAL-14Nov2018-WMS.pdf.

3. Chuchalin AG, Khaltaev N, Antonov NS, Galkin DV, Manakov LG, Antonini P, Murphy M, 
Solodovnikov AG, Bousquet J, Pereira MH, Demko IV. Chronic respiratory diseases and risk factors in 12 regions of the Russian Federation. Int J Chron Obstruct Pulmon Dis. 2014;9:963-74. doi: 10.2147/COPD.S67283.

4. Rabe KF, Hurd $S$, Anzueto A, Barnes PJ, Buist SA, Calverley P, Fukuchi Y, Jenkins C, Rodriguez-Roisin R, van Weel C, Zielinski J; Global Initiative for Chronic Obstructive Lung Disease. Global strategy for the diagnosis, management, and prevention of chronic obstructive pulmonary disease: GOLD executive summary. Am J Respir Crit Care Med. 2007;176(6):532-55. doi: 10.1164/ rccm.200703-456SO.

5. Министерство здравоохранения Российской Федерации. Российское респираторное общество. Хроническая обструктивная болезнь легких. Клинические рекомендации. 2018 [Интернет]. Доступно на: http://spulmo.ru/upload/federal_klinicheskie_rekomendaciy_hobl.pdf. [Ministry of Healthcare of Russian Federation. Russian Respiratory Society. Chronic Obstructive Lung Disease: Clinical Guidelines. 2018 [Internet]. Available from: http://spulmo.ru/ upload/federal_klinicheskie_rekomendaciy_ hobl.pdf. Russian.]

6. Burgel PR. The role of small airways in obstructive airway diseases. Eur Respir Rev. 2011;20(119):23-33. doi: 10.1183/09059180.00010410.

7. Авдеев СН. Малые дыхательные пути при хронической обструктивной болезни легких - важнейшая мишень эффективной терапии. Пульмонология. 2012;(6):11126. doi: 10.18093/0869-0189-2012-0-6111-126. [Avdeev SN. [Small airways in chronic obstructive pulmonary disease as a core target of effective therapy]. Pulmonologiya. 2012;(6):111-26. Russian. doi: 10.18093/0869-0189-2012-0-6-111-126.]

8. Hogg JC, Paré PD, Hackett TL. The contribution of small airway obstruction to the pathogenesis of chronic obstructive pulmonary disease. Physiol Rev. 2017;97(2):529-52. doi: 10.1152/physrev.00025.2015.

9. Hogg JC, Chu F, Utokaparch S, Woods R, Elliott WM, Buzatu L, Cherniack RM, Rogers RM, Sciurba FC, Coxson HO, Paré PD. The nature of small-airway obstruction in chronic obstructive pulmonary disease. N Engl J Med. 2004;350(26):2645-53. doi: 10.1056/NEJMoa032158.

10. Hogg JC, Chu FS, Tan WC, Sin DD, Patel SA, Pare PD, Martinez FJ, Rogers RM, Make BJ, Criner GJ, Cherniack RM, Sharafkhaneh A, Luketich JD, Coxson HO, Elliott WM, Sciurba FC. Survival after lung volume reduction in chronic obstructive pulmonary disease: insights from small airway pathology. Am J Respir Crit Care Med. 2007;176(5):454-9. doi: 10.1164/rccm.200612-1772OC.
11. Haruna $A$, Oga $T$, Muro $S$, Ohara $T$, Sato $S$, Marumo S, Kinose D, Terada K, Nishioka M, Ogawa E, Hoshino Y, Hirai T, Chin K, Mishima M. Relationship between peripheral airway function and patient-reported outcomes in COPD: a cross-sectional study. BMC Pulm Med. 2010;10:10. doi: 10.1186/14712466-10-10.

12. Черняк АВ. Функциональные методы диагностики патологии мелких дыхательных путей. Атмосфера. Пульмонология и аллергология. 2013;(1):36-41. [Cherniak AV. [Functional diagnostic methods for small airway disease]. Atmosfera. Pulmonologiya i allergologiya. 2013;(1):36-41. Russian.]

13. McNulty W, Usmani OS. Techniques of assessing small airways dysfunction. Eur Clin Respir J. 2014;1. doi: 10.3402/ecrj.v1.25898.

14. Bonini M, Usmani OS. The role of the small airways in the pathophysiology of asthma and chronic obstructive pulmonary disease. Ther Adv Respir Dis. 2015;9(6):281-93. doi: 10.1177/1753465815588064.

15. Айсанов ЗР, Калманова ЕН. Поражение малых дыхательных путей при бронхиальной астме: новые данные, новая парадигма. Практическая пульмонология. 2019;(1):614. [Aisanov ZR, Kalmanova EN. [The lesion of small airways in patients with asthma: new data, new paradigm]. Practical Pulmonology. 2019;(1):6-14. Russian.]

16. Miller MR, Hankinson J, Brusasco V, Burgos F, Casaburi R, Coates A, Crapo R, Enright $P$, van der Grinten $C P$, Gustafsson $P$, Jensen $R$, Johnson DC, Maclntyre N, McKay R, Navajas D, Pedersen OF, Pellegrino R, Viegi G, Wanger J; ATS/ERS Task Force. Standardisation of spirometry. Eur Respir J. 2005;26(2):319-38. doi: 10.1183/09031936.05.00034805.

17. Wanger J, Clausen JL, Coates A, Pedersen OF, Brusasco V, Burgos F, Casaburi R, Crapo $R$, Enright $P$, van der Grinten $C P$, Gustafsson $P$, Hankinson J, Jensen R, Johnson $D$, Macintyre N, McKay R, Miller MR, Navajas D, Pellegrino R, Viegi G. Standardisation of the measurement of lung volumes. Eur Respir J. 2005;26(3):511-22. doi: 10.1183/09031936.05.00035005.

18. Macintyre N, Crapo RO, Viegi G, Johnson DC, van der Grinten $C P$, Brusasco V, Burgos $F$, Casaburi R, Coates A, Enright P, Gustafsson P, Hankinson J, Jensen R, McKay R, Miller MR, Navajas $D$, Pedersen OF, Pellegrino $R$, Wanger J. Standardisation of the single-breath determination of carbon monoxide uptake in the lung. Eur Respir J. 2005;26(4):720-35. doi: 10.1183/09031936.05.00034905.

19. Smith HJ, Reinhold P, Goldman MD. Forced oscillation technique and impulse oscillometry. European Respiratory Monograph. 2005;31:72-105.

20. Wei X, Shi Z, Cui Y, Mi J, Ma Z, Ren J, Li J, Xu S, Guo Y. Impulse oscillometry system as an alternative diagnostic method for chronic obstructive pulmonary disease. Medicine (Baltimore). 2017;96(46):e8543. doi: 10.1097/ MD.0000000000008543.

21. Quanjer PH, Tammeling GJ, Cotes JE, Pedersen OF, Peslin R, Yernault JC. Lung volumes and forced ventilatory flows. Report Working Party Standardization of Lung Function Tests, European Community for Steel and Coal. Official Statement of the European Respiratory Society. Eur Respir J Suppl. 1993;16:5-40.

22. Winkler J, Hagert-Winkler A, Wirtz H, Hoheisel G. Die moderne Impulsoszillometrie im Spektrum lungenfunktioneller Messmethoden [Modern impulse oscillometry in the spectrum of pulmonary function testing methods]. Pneumologie. 2009;63(8):461-9. German. doi: 10.1055/s-0029-1214938.

23. Perez T, Chanez $P$, Dusser D, Devillier P. Small airway impairment in moderate to severe asthmatics without significant proximal airway obstruction. Respir Med. 2013;107(11):1667-74. doi: 10.1016/j. rmed.2013.08.009.

24. Oppenheimer BW, Goldring RM, Herberg ME, Hofer IS, Reyfman PA, Liautaud S, Rom WN, Reibman J, Berger KI. Distal airway function in symptomatic subjects with normal spirometry following World Trade Center dust exposure. Chest. 2007;132(4):1275-82. doi: 10.1378/chest.07-0913.

25. Contoli M, Bousquet J, Fabbri LM, Magnussen $H$, Rabe KF, Siafakas NM, Hamid $Q$, Kraft M. The small airways and distal lung compartment in asthma and COPD: a time for reappraisal. Allergy. 2010;65(2):141-51. doi: 10.1111/j.1398-9995.2009.02242.x.

26. Cosio M, Ghezzo H, Hogg JC, Corbin R, Loveland M, Dosman J, Macklem PT. The relations between structural changes in small airways and pulmonary-function tests. N Engl J Med. 1978;298(23):1277-81. doi: 10.1056/ NEJM197806082982303.

27. Manoharan A, Anderson WJ, Lipworth J, Lipworth BJ. Assessment of spirometry and impulse oscillometry in relation to asthma control. Lung. 2015;193(1):47-51. doi: 10.1007/ s00408-014-9674-6.

28. Pisi R, Aiello $M$, Zanini A, Tzani P, Paleari $D$, Marangio E, Spanevello A, Nicolini G, Chetta A. Small airway dysfunction and flow and volume bronchodilator responsiveness in patients with chronic obstructive pulmonary disease. Int J Chron Obstruct Pulmon Dis. 2015;10:1191-7. doi: 10.2147/COPD.S82509.

29. Williamson PA, Clearie K, Menzies D, Vaidyanathan S, Lipworth BJ. Assessment of small-airways disease using alveolar nitric oxide and impulse oscillometry in asthma and COPD. Lung. 2011;189(2):121-9. doi: 10.1007/s00408-010-9275-y. 
30. Crisafulli E, Pisi R, Aiello M, Vigna M, Tzani $P_{1}$ Torres A, Bertorelli G, Chetta A. Prevalence of small-airway dysfunction among COPD patients with different GOLD stages and its role in the impact of disease. Respiration. 2017;93(1):32-41. doi: 10.1159/000452479.

31. Calverley PM, Burge PS, Spencer S, Anderson JA, Jones PW. Bronchodilator reversibil- ity testing in chronic obstructive pulmonary disease. Thorax. 2003;58(8):659-64. doi: 10.1136/thorax.58.8.659.

32. Prentice HA, Mannino DM, Caldwell GG, Bush HM. Significant bronchodilator responsiveness and "reversibility" in a population sample. COPD. 2010;7(5):323-30. doi: 10.3109/15412555.2010.510161.

\title{
Diagnosis of small airway dysfunction in patients with chronic obstructive pulmonary disease
}

\author{
A.V. Cherniak' • O.I. Savushkina² -T.L. Pashkova' • E.V. Kryukov²
}

Background: Small airway dysfunction (SAD) is a functional hallmark of chronic obstructive pulmonary disease (COPD). However, SAD prevalence and its role in COPD pathophysiology are not yet sufficiently studied.

Aim: To assess the prevalence of SAD in COPD patients by various functional diagnostic methods, such as spirometry, body plethysmography, and impulse oscillometry (IOS).

Materials and methods: This was an cross-sectional study. Spirometry, body plethysmography and IOS were used in 132 COPD patients in remission under standard anti-COPD treatments. The presence of SAD was confirmed by at least one of the following criteria or their combination: 1) by spirometry: the difference between vital capacity (VC) and forced vital capacity (FVC) $>10 \%$; 2) the presence of air trapping by body plethysmography; 3) identification of the frequency dependence of the resistive resistance at 5 to $20 \mathrm{~Hz}$ (R5 - R20 > $0.07 \mathrm{kPa} \times \mathrm{s} / \mathrm{l})$, as assessed by IOS

Results: Mean forced expiratory volume in $1 \mathrm{~s}$ $\left(F V_{1}\right)$ was $42.9 \%$ of predicted. Depending on the severity of the obstruction, the patients were divided into 4 groups: 7 patients (group 1) had the obstruction corresponding to GOLD 1 stage, 37 (group 2) to GOLD 2, 49 (group 3) to GOLD 3, and 39 (group 4) to GOLD 4. SAD was found in $96 \%$ of COPD patients, whereas in those with severe obstruction (GOLD 3-4), it was present in $100 \%$ of the cases. By spirometry, SAD was identified only in $67 \%$ of COPD patients, by body plethysmography in $75 \%$ of COPD patients (in those with severe obstruction (GOLD 3 and 4 ) in 88 and 97\%, respectively). With IOS, it was possible to identify SAD in $94 \%$ of patients and in $100 \%$ of those with severe obstruction (GOLD 3-4).

Conclusion: With deterioration of obstructive pulmonary ventilation abnormalities in COPD patients, there is a progressive increase in small airway dysfunction. Impulse oscillometry seems to be the most effective method for diagnosis of small airway dysfunction, as it helped to identify SAD in $94 \%$ of COPD patients and in $100 \%$ of those with severe and very severe obstruction.

Key words: small airway dysfunction, chronic obstructive pulmonary disease, impulse oscillometry, spirometry, body plethysmography

For citation: Cherniak AV, Savushkina OI, Pashkova TL, Kryukov EV. Diagnosis of small airway dysfunction in patients with chronic obstructive pulmonary disease. Almanac of Clinical Medicine. 2020;48(5):307-15. doi: 10.18786/2072-0505-2020-48-019.

Received 29 October 2019; revised 19 November 2019; accepted 1 April 2020; published online 10 April 2020

\section{Conflict of interests}

The authors declare no obvious and potential conflicts of interests related to the publication.

\section{Authors' contributions}

A.V. Cherniak, elaboration of the project design, recruitment of the patient groups, clinical data collection, analysis and interpretation of the results, text writing; O.I. Savushkina, elaboration of the project design, recruitment of the patient groups, clinical data collection, analysis and interpretation of the results, text writing and editing; T.L. Pashkova, clinical data collection, analysis and interpretation of the results, text editing; E.V. Kryukov, the paper concept and design, text editing, approval of the final version of the manuscript. All the authors made their significant contributions to the research and preparation of the article, have read and approved the final version before submission
33. Черняк АВ, Авдеев СН, Пашкова ТЛ, Айсанов ЗР. Бронходилатационный тест у больных с хронической обструктивной болезнью легких. Пульмонология. 2003;(1):51-6. [Cherniak AV, Avdeev SN, Pashkova TL, Aysanov ZR. [Reversibility test in patients with chronic obstructive pulmonary disease]. Pulmonology. 2003;(1):51-6. Russian.]
Alexander V. Cherniak - MD, PhD, Head of the Laboratory of Functional and Ultrasonic Research Methods'; ORCID: https://orcid.org/0000-0002-2001 5504

$\triangle 28$ Orekhovyy bul'var, Moscow, 115682, Russian Federation. Tel.: +7 (917) 5500634 E-mail: achi2000@mail.ru

Olga I. Savushkina - PhD (in Biol.), Head of Department of Lung Function Testing, Center of Functional Diagnostic Investigations ${ }^{2}$; ORCID: https://orcid.org/0000-0002-7486-4990. E-mail: olga-savushkina@yandex.ru

Tatyana L. Pashkova - MD, PhD, Senior Research Fellow, Laboratory of Functional and Ultrasonic Research Methods ${ }^{1}$

E-mail: tanya.pashkova.33@mail.ru

Evgeniy V. Kryukov - MD, PhD, Professor, Corresponding Member of Russian Academy of Sciences, Head²; ORCID: https://orcid.org/0000-00028396-1936. E-mail: evgeniy.md@mail.ru
1 Pulmonology Scientific Research Institute, Federal Medical and Biological Agency of Russian Federation; 28 Orekhovyy bul'var, Moscow, 115682 Russian Federation

${ }^{2}$ N.N. Burdenko Main Military Clinical Hospital; 3 Gospital'naya ploshchad', Moscow, 105094, Russian Federation 